\title{
How biomanufacturing can save the world
}

\author{
With supply-chain shortages and production problems exacerbating vaccine inequity, global manufacturing must \\ expand to ensure the world's poorest nations are better served.
}

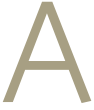

$s$ the one-year anniversary of the first Emergency Use Authorization of a COVID-19 vaccine approaches, nine in ten Africans remain unvaccinated. Manufacturing and supply-chain disruption are partly to blame, but the fault lines are deeper. Vaccine nationalism, export bans, regulatory delays, red tape, dose dumping and distribution problems have all have played their part in slowing immunizations in poorer countries. To prepare for the next outbreaks, the drug industry and international community must come together to share know-how and expand manufacturing capacity across the world.

It is 18 months into the pandemic and the suffering and health disparities continue. The United Kingdom and Singapore are in the midst of new surges, despite vaccination rates of $68 \%$ and $82 \%$, respectively. Hospitalizations and deaths are soaring in eastern Europe and Russia, where vaccination rates are low. And in Africa, from mid-June to September, the Delta variant rose to a peak of $>38,000$ cases per day, with a death rate of $3.7 \%$. As long as the virus continues to rage, the risk remains that a deadlier variant will emerge.

In previous pandemics, rich countries prioritized immunization of their own citizens over the rest of the world. To stop vaccine hoarding this time around, the World Health Organization (WHO), Gavi and the Coalition for Epidemic Preparedness Innovations set up COVAX, a vaccine facility aiming to provide 1.8 billion doses to the world's 92 poorest countries by early 2022 .

It hasn't exactly gone to plan.

High-income nations, comprising just $16 \%$ of the world's population, gobbled up $>70 \%$ of the available doses of the five leading COVID-19 vaccines. At the same time, COVAX has faltered, pledges have come up short (in September, the United States finally topped up donations to 1 billion doses), deliveries have been delayed (with only 371.1 million shipped doses so far), communication lapses have led to last-minute changes in supply volumes and timelines, batches have arrived too close to expiry dates, doses have arrived but lacked syringes to administer them, and a lack of trained personnel or cold-chain in low- and middle-income countries (LMICs) has delayed distribution. When the extent of these delays became clear, many nations opened up their own bilateral negotiations with manufacturers, further compounding the confusion. Today, COVAX projects only 1.2 billion doses will be delivered by the year's end.

In April, amidst a devastating wave of COVID-19, the Indian government shut down exports from the Serum Institute of India-one of COVAX's main suppliers, contracted to produce 1.1 billion doses, many of which were allocated to LMICs.

Other manufacturers have failed to deliver on commitments. According to analyst Airfinity, US and European vaccine makers missed 2020 and 2021 targets by $96 \%$ and $50 \%$, respectively. If Chinese producers CoronaVac and Sinopharm hadn't stepped up to provide $>1$ billion doses to poorer countries (nearly half of the global total), the situation would be even worse.

Some of the problems are understandable. Producing mRNA vaccines at global scale has never before been attempted; Moderna had to establish its entire supply chain from scratch while Pfizer had to coordinate its 86 suppliers in 19 countries to produce a 280 -component vaccine. Against a background of shortages of tubing, glass vials, pumps and components such as cholesterol, each round of scale-up has required repeated quality checks, regulatory authorizations and training on the fly. In the United States, quality-control concerns at Emergent Biosolutions led to the April shuttering of its facility and the destruction of tens of millions of vaccine doses; AstraZeneca got in a spat with the European Union over the export of doses from its Brussels facility; and South African contractor Aspen Pharmaceuticals courted international controversy when it directed doses away from Africa to fulfill European commitments.

As the frustrations grew, IP waivers and compulsory licensing were advanced as solutions. But Moderna already ceded its global patent rights. And establishing new global production facilities is not a near-term fix for vaccine shortages.

A more immediate solution is to remove export barriers-India relaxed its ban in October-and for aid agencies to improve vaccine distribution and coordination. To overcome the lack of transparency around contractual obligations and the ultimate destination of manufacturer's doses, it may also be worth establishing a central marketplace to facilitate the resale of vaccine surpluses.

That does not mean that the sharing of manufacturing expertise should be off the table. On the contrary, pandemic preparedness demands that efforts redouble to expand vaccine production capacity on every continent.

Thus far, manufacturers have announced plans to set up their own manufacturing centers in Africa, Latin America and Asia. But they have stonewalled WHO's efforts to encourage knowledge transfer via the COVID-19 Technology Access Pool (C-TAP) and Medicines Patent Pool. And they have declined to participate in WHO's mRNA manufacturing hubs, such as the one recently set up in South Africa, citing concerns that producers in the global South may compromise their vaccine brands and quality standards. Their concern is that any quality problem with a vaccine would likely compromise uptake, promote hesitancy and damage the brand.

On the other hand, these arguments are similar to ones made by brand manufacturers over a decade ago when biosimilars were first introduced. Originators labeled biosimilars as 'unsafe', claiming only they had the relevant know-how and resources to assure the quality of biologics. This has proven patently untrue. Today, many tens of companies around the world produce high-quality biosimilars.

Vaccine manufacturers have a choice to make. Either they reconsider the merits of sharing their know-how to galvanize untapped manufacturing capacity around the globe-with the reputational benefits and licensing royalties that would accrue. Or they expand their own global manufacturing capacity to reach all corners of the globe. Failure to do one or the other will simply perpetuate the grave inequities created during the 2021 vaccine rollout when the next pandemic comes around.

Published online: 27 October 2021 https://doi.org/10.1038/s41587-021-01132-x 